\title{
Application of the arm-cranking 30-second Wingate Anaerobic Test (the WAnT) to assess power in amputee football players
}

\author{
AgnieszKa Magdalena NowaK*, Bartosz Molik, ANDRZeJ Kosmol, \\ MATEUSZ SZCZEPANIAK, JOLANTA MARSZAŁEK
}

Faculty of Rehabilitation, Józef Piłsudski University of Physical Education, Warsaw, Poland.

\begin{abstract}
Purpose: The aim of this work was to determine anaerobic performance in male amputee football players considering types and levels of limb impairment, playing position, anthropometric parameters, and comparing the findings to reference values. Relationship between parameters in the laboratory anaerobic test and the handgrip test was checked. Methods: The 30-second Wingate Anaerobic Test (peak power, mean power, relative peak power, relative mean power, time to achieve peak power, fatigue index) on the arm-crank ergometer (LODE ANGIO), the FUTREX 6100 (Futrex, Gaithersburg, USA) and the handgrip test were used in amputee football players $(n=23)$. Anthropometric measurements were collected. Results: There were no differences in anaerobic results between players considering types and levels of limb impairment. Forwards had significantly higher relative mean and peak power $(p=0.049, d=$ $0.82 ; p=0.049, d=0.81)$, and lower amputation-adjusted body mass index $(p=0.001, d=1.50)$ than defenders. For peak power, 19 out 23 achieved, and for relative peak power, 22 out 23 achieved results from "average" to "elite". Peak power strongly correlated to handgrip strength results. Conclusions: Amputee football requires a high level of power from players. Maintaining appropriate body composition is important for amputee football players to have better anaerobic performance during the game. The 30-second Wingate Anaerobic Test can be used to assess anaerobic performance in AF players. Sport-specific anaerobic performance laboratory tests and field-based tests using in indirect upper limbs' peak power monitoring would be beneficial for coaches.
\end{abstract}

Key words: disability sport, soccer, adapted, physical fitness, people with special needs, classification

\section{Introduction}

Amputee football (amputee soccer, AF) is a variety of soccer game designed for individuals with limb impairments [6]. This team game is becoming increasingly popular not only thanks to able-bodied soccer popularity. It also constitutes a good example of part of a rehabilitation process which helps people with lower-limb deficiency and leg length differences to maintain a high level of physical functionality. The rules are similar to able-bodied soccer with few exceptions, e.g., athletes with lower-limb impairments move on a field using forearm type crutches (field players), whereas athletes with upper limb impairments must keep inside the body of the jersey and bandage this limb (goalkeepers). Artificial limbs and prostheses need to be removed before the game starts. It is prohibited to use the impaired limb to control or direct the ball. The match is played for two halves of 25 minutes each, on a smaller field $(60 \times 40 \mathrm{~m})$ by seven players from each team (7-a-side soccer). A maximum of 2 substitutes may enter the game at any time and all substituted players are allowed to return to the game to replace any player on the field of play [6], [40].

The observations of AF matches demonstrate that players move all the time on the field without stop.

\footnotetext{
* Corresponding author: Agnieszka Magdalena Nowak, Józef Piłsudski University of Physical Education, ul. Marymoncka 34, 00-968 Warsaw, Poland. Phone: +48516566280, e-mail: agnieszka.nowak@awf.edu.pl

Received: February 10th, 2021

Accepted for publication: May 26th, 2021
} 
When AF players try to catch a ball, they get into the position or play one-by-one. In that situation they move extraordinarily fast for fractions of a second and this move last merely a few seconds (short-term efforts) [15]. They need to be physically well prepared to accelerate and slow down at short notice, concentrate and control a ball at the same time, and react on anticipated opponent's move. Both aerobic and anaerobic performance are important in this discipline. Players perform high-intensity efforts (i.e., average HR of $81-96 \%$ HRmax) and cover 2-6 km during each game, although high-speed runs and sprints (above 13 or $15 \mathrm{~km} / \mathrm{h}$ ) take about $2-27 \%$ of playing time [15], [26], [32], anaerobic performance appears to be a particularly important ability for AF players because it generates actions and competitive advantage that might significantly contribute to winning a game [8], [27]. AF players involved use both upper and lower limbs and it is more taxing than in able-bodied soccer [34]. They need a good technique of moving by crutches to do fast and dynamic starts and changes of directions. AF players have specific move pattern, i.e., when running, at first, they put crutches on the ground using strong push of upper limbs, secondly, they put healthy lower limb and subsequently jump on it and again put crutches. It requires from them a great amount of strength, and power of upper limbs to move throughout the whole match, as the load on upper limbs varies between 111 and $175 \%$ of whole-body weight (WBW) [34]. Thys et al. [35] found that crutch walking requires 2-3 times more energy than a two-leg swing-through gait pattern (this difference was found between trained and after a sport trauma participants). Perhaps, running with crutches needs more energy compared to crutch walking. Moreover, fatigue experienced after AF matches might lead to impairments in muscular performance, especially for upper limbs [32]. Also, a significantly higher reduction in upper limb muscle endurance and lower-limb power can be observed after a match $(-20 \% ; p=0.01$ and $-5 \% ; p=0.02$, respectively), with no decrease in physical match performance [32]. It is important to note that muscle mass of upper limbs is far lesser than the one of lower limbs. As a consequence, upper limbs require much more energy expenditure to generate similar power. It can be presumed that $\mathrm{AF}$ is full of intermittent high-intensity actions interspersed with short recovery periods and belongs to an aerobic-anaerobic (stop-go) type of sport like able-bodied soccer [27], [33], [40]. Nevertheless, there are no thorough studies on muscles' involvement in $\mathrm{AF}$ match performance. It can be hypothesized that even higher-intensity short efforts (anaerobic performance) are observed among these players but to date, no in-depth analysis related to this issue has been carried out.

The analysis demonstrates insufficient research on anaerobic performance in AF which relates exclusively to game-induced muscular fatigue, match impact on muscular power and strength, sprint performance and effects of playing AF on physical fitness [9], [19], [27], [31], [32], [38]. While the researchers have used outfield tests such as countermovement jump, medicine ball throw, sprint test, handgrip test, there are no references to validity in amputee population, nor the appropriate tests to assess anaerobic performance in AF players.

Taking the above-mentioned factors into account, it was decided to take advantage of the 30 -second Wingate Anaerobic Test (the WAnT), which proves to be valid, reliable, safe and easy-to-perform for athletes [11] and is widely applied among athletes with impairments, i.e., by wheelchair rugby players, wheelchair basketball players or sitting volleyball players [5], [10], [16], [17], [21]-[23]. This test engages upper limbs muscles, and in our opinion, it is appropriate and relevant to start AF players' anaerobic performance assessment.

As it was mentioned earlier, anaerobic performance plays a determinant role in AF. The importance of WBW and body composition in anaerobic performance is presented in some manuscripts [19], [27]. There are six field players similar in terms of functionality (lower-limb impairment), but the level and the type of impairment may have influence on anaerobic performance and physical fitness as in other parasports disciplines [2], [16]. Players' playing positions may be directly connected and influenced by their impairment. It is proven that the energy cost of physical activities is higher in persons with an above knee amputation compared to those with below knee amputations [37]. It is not clear whether the level of amputation or type of impairment have influence on players' game performance in AF. Emphasizing the importance of anaerobic performance in this sport discipline and probability of difference in players' performance due to their impairment, the study sought to determine anaerobic performance in male AF players in terms of the type and the level of limb impairment, playing position, and anthropometric parameters, and comparing the findings with reference values for athletes with lower-limb impairments [20]. Moreover, it was verified if there was a relationship between parameters in the WAnT and the handgrip test conducted among AF players. 


\section{Materials and methods}

\subsection{Participants}

Twenty-five Polish national male AF players were invited to participate in this study. The selection criteria were as follows: gender (males only), lower-limb impairments, retained hands, grip function and a minimum of six months of training experience. Goalkeepers were excluded due to their upper limb impairments and limited handgrip function.

Each participant was tested individually and with due diligence. First, they were informed about the procedures, benefits, and risks of the investigation as well as about the possibility to withdraw from the study without any consequences. Each participant signed an informed consent.

The study was approved by the Institutional Review Board (the Local Ethics Committees; SKE 01-53/2017) and it conformed to the Declaration of Helsinki.

\subsection{Procedures}

This study was performed in one day, during the camp of the Polish national AF team. The order of actions was as follows: completing the survey (1), performing anthropometric measurements (2), handgrip strength measurements (3), and the WAnT (4).

\subsubsection{The survey (1)}

Each participant completed the survey containing personal information, such as: age, types of lowerlimb impairment (limb deficiency or leg length difference), level of leg length, training experience and playing position. Limb deficiency is "total or partial absence of bones or joints as a consequence of trauma (e.g., car accident), illness (e.g., bone cancer) or congenital limb deficiency (e.g., dysmelia)" (IPC, $2015,2016)$ and applies to amputees [12], [13]. Leg length difference is "bone shortening in one leg due to congenital deficiency or trauma" (IPC, 2015, 2016) and applies to individuals with one limb shorter than the other [12], [13]. Declared levels of leg length were verified by the investigators, who were physiotherapists.

\subsubsection{Anthropometric measurements (2)}

WBW and body height measurements were carried out on a weighting scale with height rod. The pros- theses were removed. Body fat percentage (FAT \%) was estimated in near-infrared (NIR) spectroscopy by FUTREX 6100 (Futrex, Gaithersburg, USA) according to the procedures recommended by the manufacturer [7] i.e., in a sitting position, in the middle distance of biceps brachii of the right arm. Amputationadjusted body mass index (A-BMI) was calculated using a formula described by other researchers [36], [39].

\subsubsection{Handgrip strength measurement (3)}

The participants performed the handgrip test in a sitting position with the tested upper limb extended along the torso and not touching the chair and torso [14], [17]. The test was performed twice for the right and the left limb. Means of the best results [Newtons; N] for each of the hands were taken into consideration. The test was performed using the hydraulic hand dynamometer (Model SH5001, Saehan Corp., Masan, Korea).

\subsubsection{Anaerobic performance test (4)}

The WAnT was performed on the arm-crank ergometer (LODE ANGIO, Groningen, Netherlands, Software Package-Wingate v.1.07b). The procedure of anaerobic performance assessment for upper limbs introduced by Inbar was used [11]. The ergometer was set on the wall and was mounted on the gymnastic ladder in the gym. The axis was set individually to the level of each participant's shoulder joints. The test was performed in a sitting position on a chair with backrest. The participants were instructed not to brace their lower limb against the stand and not to lean on the backrest. There were no straps supporting the stump and the lower limb. The load was calculated individually for each player based on his WBW (5\% of the body mass), and automatically dosed by a computer program [24]. The athletes started with a 2-minute warm-up on the ergometer with $50 \mathrm{~W}$ load. During this period, the participants learned the specifics of movement and resistance, and adopted the most comfortable position. Then, they were asked to perform the test as fast as they could for 30 seconds and were being verbally supported throughout the test. Six parameters were observed in the WAnT: peak power (PP; defined as the highest 5-s power output), mean power (MP; defined as the average power sustained throughout the $30-\mathrm{s}$ period), relative peak power (rPP; scaled to individual body mass in kilograms), relative mean power (rMP; scaled to individual body mass in kilograms), time to achieve peak power (tPP) and fatigue index 
(FI; defined as the difference between PP and the lowest power output during the test in $\mathrm{W} / \mathrm{s}$ ) [11], [17]. The participants were monitored after the test, and they were asked to keep performing the movement on the arm-crank ergometer with no resistance as a cool-down time.

\subsection{Statistical analysis}

Statistical analysis was performed using IBM SPSS Statistics 25. In the statistical analysis, the values of such parameters as age [years], WBW $[\mathrm{kg}]$, body height [cm], FAT [\%], A-BMI, PP [W], MP [W], rPP [W/kg], rMP [W/kg], tPP [s], FI [W/s] and handgrip strength $[\mathrm{N}]$ were compared with the types of impairment (two groups: limb deficiency and leg length difference), level of leg length (two groups: AKA (impaired lower-limb shorter than the opposite thigh, e.g., above knee amputation and similar functional level of leg length) and BKA (impaired lower-limb longer than the opposite thigh, e.g., below knee amputation and similar functional level of leg length)) and playing positions (two groups: defenders and forwards with strikers and midfielders). The data distribution was verified by the Kolmogorov-Smirnov test. Not all variables were normally distributed $(p<0.05)$, therefore, the Spearman's rho $\left(r_{s}\right)$ correlation coefficient test was used to investigate the correlation between anthropometric results, handgrip test results and anaerobic performance parameters. The level of statistical significance was set at $p<0.05$. The level of correlation was assumed as moderate $(0.4<|r| \leq 0.7)$, strong $(0.7<|r|<1)$ or full $(|r|=1)$ [1]. The equality of variance was checked with the Levene's test. The $T$-test and the Mann-Whitney $U$-test were applied for independent samples to calculate the differences between the groups. Moreover, the Effect Size (ES) was displayed with Cohen's $d: d=\left|\left(\bar{x}_{1}-\bar{x}_{2}\right) / \mathrm{SD}\right|$ where small ES is when $d$ is $0.20-0.49$, medium when $d$ is $0.50-0.79$, and large - when $d$ is above 0.80 [3]. Then, the obtained results were compared to reference values developed for athletes with lower-limb impairments (group C). Group C consisted of 134 athletes representing different Paralympic sports $(31.1 \pm 10.2$ years, $77.3 \pm 12.5 \mathrm{~kg}, 8.3 \pm 6.9$ years of training experience). The athletes had lower-limb impairment such as lower-limb amputation and paresis. As AF players, they were capable of walking (they did not use wheelchairs). Results were demonstrated with regard to three age groups (I - 18-25 years, II - 26-
35 years, III - above 35 years) for each parameter [20].

\section{Results}

Twenty-three Polish male AF players took part in this study $(28.0 \pm 7.5$ years, $73.0 \pm 14.8 \mathrm{~kg}, 176.7$ $\pm 6.9 \mathrm{~cm})$. Goalkeepers were excluded because of the exclusion criteria. The characteristics of the study group are presented in Table 1.

Table 1. Characteristic of the study group $(n=23)$

\begin{tabular}{|l|c|c|}
\hline \multicolumn{1}{|c|}{ Parameter } & $\bar{x}$ & SD \\
\hline Age [years] & 28.0 & 7.5 \\
\hline Body height [cm] & 176.7 & 6.9 \\
\hline WBW [kg] & 73.0 & 14.8 \\
\hline A-BMI & 24.8 & 4.6 \\
\hline FAT \% [\%] & 14.2 & 6.2 \\
\hline Training experience [years] & 2.2 & 1.4 \\
\hline & $n$ & $\%$ \\
\hline Impairment status & & \\
\hline Leg length difference & 7 & $(30)$ \\
\hline Limb deficiency & 16 & $(70)$ \\
\hline Level of leg length status & & \\
\hline AKA & 11 & $(48)$ \\
\hline BKA & 12 & $(52)$ \\
\hline Playing position status & & \\
\hline Defenders & 10 & $(43)$ \\
\hline Forwards & 13 & $(57)$ \\
\hline
\end{tabular}

WBW - whole-body weight; A-BMI - amputation-adjusted BMI; FAT \% - percentage of body fat; AKA - impaired lowerlimb shorter than the opposite thigh and a similar functional level of leg length; BKA - impaired lower-limb longer than the opposite thigh and a similar functional level of leg length.

\subsection{Relationships between anthropometric, handgrip strength and anaerobic performance parameters into the types of impairment}

In conducted analysis, whereby the participants were divided according to the types of impairment, a significant difference in FI $(p=0.026, d=0.72)$ (Table 2) was observed. According to baseline [20], players achieved "above average" score in rPP and "average" scores in PP, rMP and MP (B group "below average" in MP) regardless of the type of impairment. 
Table 2. Differences in anthropometric, handgrip strength and anaerobic performance parameters

between the leg length difference group (A: $n=7)$ and the limb deficiency group (B: $n=16)$

\begin{tabular}{|c|c|c|c|c|c|c|}
\hline & & $\bar{x}$ & SD & Min-Max & $p$ & $d$ \\
\hline \multirow{2}{*}{ Age [years] } & A & 23.43 & 5.13 & $17.00-29.00$ & \multirow{2}{*}{$0.052^{\mathrm{T}}$} & \multirow{2}{*}{ n.s } \\
\hline & $\mathrm{B}$ & 30.00 & 7.68 & $17.00-47.00$ & & \\
\hline \multirow{2}{*}{$\begin{array}{l}\text { Body } \\
\text { height }[\mathrm{cm}]\end{array}$} & A & 180.43 & 5.65 & $170.00-187.00$ & \multirow{2}{*}{$0.087^{\mathrm{T}}$} & \multirow{2}{*}{ n.s. } \\
\hline & $\mathrm{B}$ & 175.06 & 6.94 & $165.00-192.00$ & & \\
\hline \multirow{2}{*}{ WBW [kg] } & A & 68.80 & 9.98 & $50.00-81.10$ & \multirow{2}{*}{$0.298^{\mathrm{T}}$} & \multirow{2}{*}{ n.s } \\
\hline & $\mathrm{B}$ & 74.76 & 16.37 & $50.00-98.00$ & & \\
\hline \multirow{2}{*}{ A-BMI } & A & 22.14 & 3.71 & $15.40-26.70$ & \multirow{2}{*}{$0.062^{\mathrm{T}}$} & \multirow{2}{*}{ n.s. } \\
\hline & B & 26.01 & 4.55 & $19.50-34.40$ & & \\
\hline \multirow{2}{*}{ FAT $\%[\%]$} & A & 11.81 & 5.80 & $3.20-18.50$ & \multirow{2}{*}{$0.230^{\mathrm{T}}$} & \multirow{2}{*}{ n.s. } \\
\hline & $\mathrm{B}$ & 15.26 & 6.30 & $4.30-25.80$ & & \\
\hline \multirow{2}{*}{$\begin{array}{l}\text { Handgrip } \\
\text { strength [N] }\end{array}$} & A & 531.54 & 62.33 & $467.94-601.85$ & \multirow{2}{*}{$0.689^{\mathrm{T}}$} & \multirow{2}{*}{ n.s. } \\
\hline & $\mathrm{B}$ & 554.53 & 117.07 & $360.31-778.40$ & & \\
\hline \multirow{2}{*}{ MP [W] } & A & 327.09 & 78.04 & $197.00-454.30$ & \multirow{2}{*}{$0.640^{\mathrm{U}}$} & \multirow{2}{*}{ n.s. } \\
\hline & B & 327.61 & 66.46 & $238.00-466.40$ & & \\
\hline \multirow{2}{*}{$\mathrm{PP}[\mathrm{W}]$} & A & 518.00 & 84.65 & $338.00-603.00$ & \multirow{2}{*}{$0.248^{\mathrm{T}}$} & \multirow{2}{*}{ n.s. } \\
\hline & $\mathrm{B}$ & 577.12 & 118.27 & $363.00-795.00$ & & \\
\hline \multirow{2}{*}{$\mathrm{tPP}[\mathrm{s}]$} & A & 5.57 & 1.28 & $4.00-7.40$ & \multirow{2}{*}{$0.500^{\mathrm{U}}$} & \multirow{2}{*}{ n.s. } \\
\hline & $\mathrm{B}$ & 5.41 & 2.20 & $3.40-12.80$ & & \\
\hline \multirow{2}{*}{$\mathrm{rMP}[\mathrm{W} / \mathrm{kg}]$} & A & 4.70 & 0.62 & $3.90-5.90$ & \multirow{2}{*}{$0.435^{\mathrm{T}}$} & \multirow{2}{*}{ n.s. } \\
\hline & $\mathrm{B}$ & 4.46 & 0.70 & $3.10-5.70$ & & \\
\hline \multirow{2}{*}{$\mathrm{rPP}[\mathrm{W} / \mathrm{kg}]$} & A & 7.60 & 0.75 & $6.50-8.50$ & \multirow{2}{*}{$0.472^{\mathrm{T}}$} & \multirow{2}{*}{ n.s. } \\
\hline & $\mathrm{B}$ & 7.95 & 1.15 & $5.80-10.20$ & & \\
\hline $\mathrm{FI}[\mathrm{W} / \mathrm{s}]$ & A & 12.90 & 2.16 & $9.20-15.80$ & $0026 * 1$ & 0.73 \\
\hline & $\mathrm{B}$ & 16.42 & 4.89 & $7.40-24.20$ & & \\
\hline
\end{tabular}

A - leg length difference group; B - limb deficiency group WBW - whole-body weight; A-BMI - amputation-adjusted BMI; FAT $\%$ - percentage of body fat; PP - peak power; MP - mean power; rPP - relative peak power; rMP - relative mean power, tPP - time to achieve peak power; FI - fatigue index; $\bar{x}$ - mean; $\mathrm{SD}$ - standard deviation; ${ }^{\mathrm{T}}-T$-test; ${ }^{\mathrm{U}}-$ Mann-Whitney $U$-test; $\mathrm{dS}^{\sim}$ - Cohen's $d$; * statistically significant difference $(p<0.05)$; n.s. - statistically not significant.

\subsection{Relationships between anthropometric, handgrip strength and anaerobic performance parameters into level of limb impairment}

Comparing the above knee amputation (AKA) to below knee amputation (BKA) groups, there were no differences between groups in the handgrip test, the WAnT and in anthropometric results $(p>0.05)(\mathrm{Ta}-$ ble 3). Anaerobic performance results in these groups were identical with the A and B groups in PP, rMP and MP (C group "below average" in MP). AKA players had better results ("above average" score) than BKA players in rPP.
Table 3. Differences in anthropometric, handgrip strength and anaerobic performance parameters between the level of leg length groups:

AKA (C: $n=11)$ and BKA (D: $n=12)$

\begin{tabular}{|c|c|c|c|c|c|c|}
\hline & & $\bar{x}$ & SD & Min-Max & $p$ & $d$ \\
\hline \multirow{2}{*}{ Age [years] } & & 30.00 & 7.14 & $23.00-47.00$ & \multirow{2}{*}{$0.232^{\mathrm{T}}$} & \multirow[b]{2}{*}{ n.s } \\
\hline & $\mathrm{D}$ & 26.17 & 7.73 & $17.00-43.00$ & & \\
\hline \multirow{2}{*}{$\begin{array}{l}\text { Body } \\
\text { height }[\mathrm{cm}]\end{array}$} & $\mathrm{C}$ & 175.36 & 5.39 & $165.00-183.00$ & \multirow{2}{*}{$0.390^{\mathrm{T}}$} & \multirow{2}{*}{ n.s } \\
\hline & $\mathrm{D}$ & 177.92 & 8.13 & $165.00-192.00$ & & \\
\hline \multirow{2}{*}{ WBW [kg] } & $\mathrm{C}$ & 73.01 & 17.56 & $50.00-98.00$ & \multirow{2}{*}{$0.983^{\mathrm{T}}$} & \multirow{2}{*}{ n.s } \\
\hline & $\mathrm{D}$ & 72.88 & 12.46 & $50.00-96.00$ & & \\
\hline \multirow{2}{*}{ A-BMI } & $\mathrm{C}$ & 25.96 & 5.45 & $19.50-34.40$ & \multirow{2}{*}{$0.267^{\mathrm{T}}$} & \multirow{2}{*}{ n.s } \\
\hline & $\mathrm{D}$ & 23.79 & 3.58 & $15.40-28.70$ & & \\
\hline \multirow{2}{*}{ FAT \% [\%] } & & 13.85 & 7.21 & 4.30 & \multirow{2}{*}{$0.799^{\mathrm{T}}$} & \\
\hline & $\mathrm{D}$ & 14.54 & 5.49 & $3.20-20.50$ & & \\
\hline \multirow{2}{*}{$\begin{array}{l}\text { Handgrip } \\
\text { strength }[\mathrm{N}]\end{array}$} & $\mathrm{C}$ & 578.71 & 69.35 & $433.70-642.68$ & \multirow{2}{*}{$0.291^{\mathrm{T}}$} & \multirow{2}{*}{ n.s } \\
\hline & $\mathrm{D}$ & 522.95 & 119.01 & $360.31-778.40$ & & \\
\hline \multirow{2}{*}{$\mathrm{MP}[\mathrm{W}]$} & $\mathrm{C}$ & 322.70 & 57.29 & $238.00-419.25$ & \multirow{2}{*}{$0.829^{\mathrm{U}}$} & \multirow{2}{*}{ n.s } \\
\hline & $\mathrm{D}$ & 331.81 & 79.49 & $197.00-466.40$ & & \\
\hline \multirow{2}{*}{$\mathrm{PP}[\mathrm{W}]$} & $\mathrm{C}$ & 591.18 & 110.37 & $425.00-795.00$ & \multirow{2}{*}{$0.190^{\mathrm{T}}$} & \multirow{2}{*}{ n.s } \\
\hline & $\mathrm{D}$ & 529.75 & 107.18 & $338.00-716.00$ & & \\
\hline \multirow{2}{*}{$\mathrm{tPP}[\mathrm{s}]$} & $\mathrm{C}$ & 5.67 & 2.59 & $3.40-2.80$ & \multirow{2}{*}{$0.926^{\mathrm{U}}$} & \multirow{2}{*}{ n.s } \\
\hline & $\mathrm{D}$ & 5.27 & 1.15 & $3.60-7.40$ & & \\
\hline \multirow{2}{*}{$\mathrm{rMP}[\mathrm{W} / \mathrm{kg}]$} & $\mathrm{C}$ & 4.55 & 0.77 & $3.10-5.70$ & \multirow{2}{*}{$0.921^{\mathrm{T}}$} & \multirow{2}{*}{ n.s } \\
\hline & $\mathrm{D}$ & 4.52 & 0.60 & $3.70-5.90$ & & \\
\hline \multirow{2}{*}{$\mathrm{rPP}[\mathrm{W} / \mathrm{kg}]$} & $\mathrm{C}$ & 8.28 & 1.12 & $6.80-10.20$ & \multirow{2}{*}{$0.051^{\mathrm{T}}$} & \multirow{2}{*}{ n.s } \\
\hline & $\mathrm{D}$ & 7.44 & 0.82 & $5.80-8.50$ & & \\
\hline $\mathrm{F}[\mathrm{W} / \mathrm{s}]$ & $\mathrm{C}$ & 17.16 & 4.27 & $9.20-24.20$ & & \\
\hline & $\mathrm{D}$ & 13.69 & 4.21 & $7.40-21.50$ & & \\
\hline
\end{tabular}

C - AKA group: impaired lower-limb shorter than the opposite thigh and a similar functional level of leg length; D - BKA group: impaired lower-limb longer than the opposite thigh and a similar functional level of leg length; WBW - whole-body weight; A-BMI - amputation-adjusted BMI; FAT \% - percentage of body fat; PP - peak power; MP - mean power; rPP - relative peak power; rMP - relative mean power, tPP - time to achieve peak power; FI - fatigue index; BKA - below knee amputation; AKA - above knee amputation; $\bar{x}$ - mean; SD - standard deviation; ${ }^{\mathrm{T}}-T$-test; ${ }^{\mathrm{U}}-$ Mann-Whitney $U$-test; $\mathrm{dS}{ }^{2}-$ Cohen's $d$; * statistically significant difference $(p<0.05) ;$ n.s. - statistically not significant.

\subsection{Relationships between anthropometric, handgrip strength and anaerobic performance parameters into different field positions}

Dividing by playing positions, forwards had significantly lower WBW ( $p=0.001, d=1.50)$, FAT $\%$ $(p=0.001, d=1.26)$, and A-BMI $(p=0.001, d=1.50)$ in comparison with defenders (the ES was large). Moreover, playing position significantly differenti- 
ated participants in $\mathrm{rMP}(p=0.049, d=0.82)$ and $\mathrm{rPP}$ ( $p=0.049, d=0.81$ ) in favour of the forwards (the ES was large). On the other hand, defenders had significantly greater PP compared to forward players $(p=0.027, d=0.91)$ (Table 4). Comparing results in the WAnT with the reference values, it turned out that forward players in rPP exceeded with "excellent" result, and defenders scored "average". Despite the type and the level of limb impairment, players had "average" result in PP, nonetheless, assessing different playing positions, defenders had better result and it was "above average". In all analysing groups the MP and rMP were on "average" or "below average" level according to reference values (Suppl. 1).

Table 4. Differences in anthropometric, handgrip strength and anaerobic performance parameters between the defenders' group (E: $n=10)$ and the forwards' group (F: $n=13$ )

\begin{tabular}{|c|c|c|c|c|c|c|}
\hline & & $\bar{x}$ & SD & Min-Max & $p$ & $d$ \\
\hline \multirow{2}{*}{ Age [years] } & $\mathrm{E}$ & 31.10 & 7.08 & $24.00-47.00$ & \multirow{2}{*}{$0.084^{\mathrm{T}}$} & \multirow{2}{*}{ n.s. } \\
\hline & $\mathrm{F}$ & 25.62 & 7.25 & $17.00-43.00$ & & \\
\hline \multirow{2}{*}{$\begin{array}{l}\text { Body } \\
\text { height }[\mathrm{cm}]\end{array}$} & $\mathrm{E}$ & 178.00 & 6.82 & $165.00-192.00$ & \multirow{2}{*}{$0.441^{\mathrm{T}}$} & \multirow{2}{*}{ n.s. } \\
\hline & $\mathrm{F}$ & 175.69 & 7.11 & $165.00-187.00$ & & \\
\hline \multirow{2}{*}{ WBW [kg] } & $\mathrm{E}$ & 85.48 & 10.77 & $63.00-98.00$ & \multirow{2}{*}{$0.001^{* \mathrm{~T}}$} & \multirow{2}{*}{$1.50^{\circ}$} \\
\hline & $F$ & 63.31 & 8.98 & $50.00-77.00$ & & \\
\hline \multirow{2}{*}{ A-BMI } & $E$ & 28.73 & 3.34 & $23.90-34.40$ & \multirow{2}{*}{$0.001^{*} \mathrm{~T}$} & \multirow{2}{*}{1.50} \\
\hline & $\mathrm{F}$ & 21.83 & 2.83 & $15.40-25.00$ & & \\
\hline \multirow{2}{*}{ FAT \% [\%] } & $\mathrm{E}$ & 18.64 & 3.87 & $13.80-25.80$ & \multirow{2}{*}{$0.001^{* \mathrm{~T}}$} & \multirow{2}{*}{1.26} \\
\hline & $\mathrm{F}$ & 10.81 & 5.58 & $3.20-19.50$ & & \\
\hline \multirow{2}{*}{$\begin{array}{l}\text { Handgrip } \\
\text { strength }[\mathrm{N}]\end{array}$} & $\mathrm{E}$ & 581.85 & 121.68 & $360.31-778.40$ & \multirow{2}{*}{$0.182^{\mathrm{T}}$} & \multirow{2}{*}{ n.s. } \\
\hline & $\mathrm{F}$ & 512.85 & 67.33 & $429.23-601.85$ & & \\
\hline \multirow{2}{*}{$\mathrm{MP}[\mathrm{W}]$} & $\mathrm{E}$ & 359.58 & 66.36 & $270.90-466.40$ & \multirow{2}{*}{$0.051^{\mathrm{U}}$} & \multirow{2}{*}{ n.s. } \\
\hline & $\mathrm{F}$ & 302.74 & 61.18 & $197.00-454.30$ & & \\
\hline \multirow{2}{*}{ PP [W] } & $\mathrm{E}$ & 615.90 & 123.06 & $363.00-795.00$ & \multirow{2}{*}{$0.027^{* \mathrm{~T}}$} & \multirow{2}{*}{0.91} \\
\hline & $\mathrm{F}$ & 515.46 & 79.85 & $338.00-620.00$ & & \\
\hline \multirow{2}{*}{$\mathrm{tPP}[\mathrm{s}]$} & $\mathrm{E}$ & 5.08 & 1.23 & $3.40-7.20$ & \multirow{2}{*}{$0.639^{\mathrm{U}}$} & \multirow[b]{2}{*}{ n.s. } \\
\hline & $\mathrm{F}$ & 5.75 & 2.35 & $4.00-12.80$ & & \\
\hline \multirow{2}{*}{$\mathrm{rMP}[\mathrm{W} / \mathrm{kg}]$} & $\mathrm{E}$ & 4.22 & 0.66 & $3.10-5.30$ & \multirow{2}{*}{$0.049^{* T}$} & \multirow{2}{*}{0.82} \\
\hline & $\mathrm{F}$ & 4.77 & 0.60 & $3.90-5.90$ & & \\
\hline \multirow{2}{*}{$\mathrm{rPP}[\mathrm{W} / \mathrm{kg}]$} & $E$ & 7.36 & 0.88 & $5.80-8.90$ & \multirow{2}{*}{$0.049^{* \mathrm{~T}}$} & \multirow{2}{*}{0.81} \\
\hline & $\mathrm{F}$ & 8.21 & 1.03 & $6.80-10.20$ & & \\
\hline $\mathrm{FI}[\mathrm{W} / \mathrm{s}]$ & $\mathrm{E}$ & 16.47 & 4.86 & $7.40-24.20$ & $0308^{\mathrm{T}}$ & \\
\hline & $\mathrm{F}$ & 14.49 & 4.20 & $9.20-22.20$ & & n. \\
\hline
\end{tabular}

E - defenders' group; F - forwards' group; WBW - whole-body weight; A-BMI - amputation-adjusted BMI; FAT \% - percentage of body fat; PP - peak power; MP - mean power; rPP - relative peak power; rMP - relative mean power, tPP - time to achieve peak power; FI - fatigue index; $\bar{x}$ - mean; SD - standard deviation; ${ }^{\mathrm{T}}-T$-test; ${ }^{\mathrm{U}}-\mathrm{Mann}$-Whitney $U$-test; $\mathrm{dS}{ }^{\vee}-$ Cohen's $d$; *statistically significant difference $(p<0.05)$; n.s. - statistically not significant.

\subsection{Results of correlations for anthropometric results, handgrip test results and anaerobic performance parameters}

Correlations between variables of anthropometric results, handgrip test results, and WAnT results are presented in Table 5. It was observed that AF players with greater WBW had statistically greater FAT \% and A-BMI, and achieved statistically higher PP values (strong correlations) and MP values (moderate correlation). A-BMI was strongly correlated with FAT \%, and moderate correlated with MP, PP, FI, and WBW. FI was strongly correlated with PP and moderately correlated with WBW, A-BMI, handgrip strength and rPP. Participants with lower FAT \% had significantly better rPP results (moderate correlation). Handgrip strength was notably positively correlated with PP. WBW, body height, A-BMI and FAT \% had significantly moderate influence on MP among AF players (Table 5).

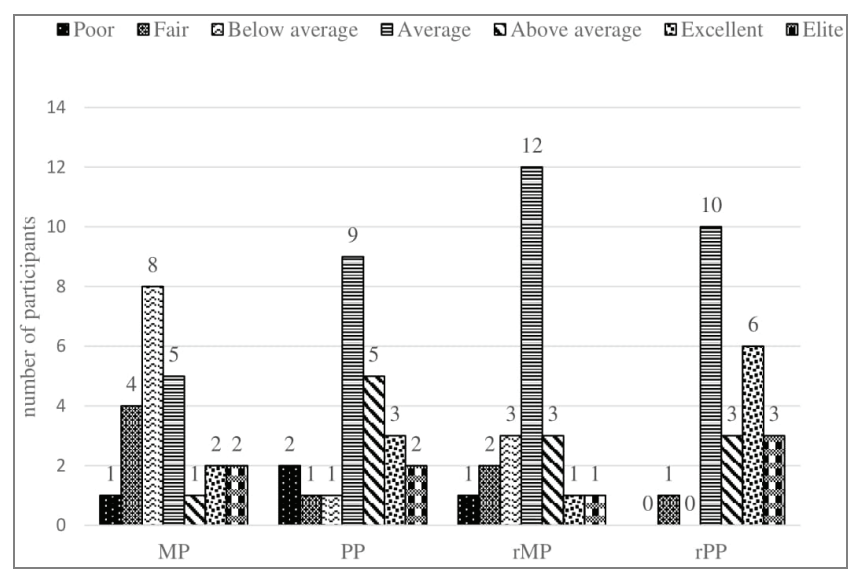

Fig. 1. AF players' results in the WanT in relation to the reference values for athletes with lower-limb impairments - group C [20]

In Figure 1, AF players' results in the WAnT in relation to the reference values for athletes with lowerlimb impairments (group C) are illustrated [20]. It is also shown the number of players reaching each level of MP, PP, rMP and rPP are also shown. AF players got better results in relative values of power (rMP, rPP). Twenty-two out of 23 players had average or better results in rPP, and 17 of them in rMP. MP was the worst parameter for AF players, in which 5 players had results above average and most of them (13 athletes) had results below average. References values are presented in Supplement 1. 
Table 5. Spearman's rho $\left(r_{s}\right)$ correlation coefficient for anthropometric results, handgrip test results and anaerobic performance parameters in amputee football players

\begin{tabular}{|c|c|c|c|c|c|c|c|c|c|c|c|c|c|}
\hline & & $\begin{array}{c}\text { Age } \\
\text { [years] }\end{array}$ & $\begin{array}{c}\text { Body } \\
\text { height } \\
{[\mathrm{cm}]}\end{array}$ & $\begin{array}{c}\text { WBW } \\
{[\mathrm{kg}]}\end{array}$ & A-BMI & $\begin{array}{c}\text { FAT \% } \\
{[\%]}\end{array}$ & $\begin{array}{l}\text { Handgrip } \\
\text { strength } \\
{[\mathrm{N}]}\end{array}$ & $\begin{array}{l}\text { MP } \\
{[\mathrm{W}]}\end{array}$ & $\mathrm{PP}[\mathrm{W}]$ & tPP $[s]$ & $\begin{array}{c}\mathrm{rMP} \\
{[\mathrm{W} / \mathrm{kg}]}\end{array}$ & $\begin{array}{c}\mathrm{rPP} \\
{[\mathrm{W} / \mathrm{kg}]}\end{array}$ & $\begin{array}{c}\mathrm{FI} \\
{[\mathrm{W} / \mathrm{s}]}\end{array}$ \\
\hline $\begin{array}{l}\text { Age } \\
\text { [years] }\end{array}$ & $r_{s}$ & & 0.057 & $0.435^{*}$ & $0.436^{* \dagger}$ & 0.346 & 0.259 & 0.258 & 0.380 & 0.043 & -0.110 & -0.016 & 0.324 \\
\hline $\begin{array}{l}\text { Body } \\
\text { height } \\
{[\mathrm{cm}]}\end{array}$ & $r_{s}$ & & 1 & $0.465 *$ & 0.079 & 0.035 & $0.801 * * \ddagger$ & $0.434 *^{\dagger}$ & $0.459^{* \dagger}$ & -0.093 & -0.044 & -0.041 & 0.174 \\
\hline $\begin{array}{l}\text { WBW } \\
{[\mathrm{kg}]}\end{array}$ & $r_{s}$ & & & 1 & $0.905^{* *} \sharp$ & $0.776^{* *} \star$ & $0.723 * *$ & $0.665^{* * \dagger}$ & $0.766^{* *} \ddagger$ & -0.080 & -0.336 & -0.269 & $0.487^{* \dagger}$ \\
\hline A-BMI & $r_{s}$ & & & & 1 & $0.906^{* *}$ & 0.433 & $0.560 * * \dagger$ & $0.678 * * \dagger$ & -0.180 & -0.384 & -0.296 & $0.460^{* \dagger}$ \\
\hline $\begin{array}{l}\text { FAT \% } \\
{[\%]}\end{array}$ & $r_{s}$ & & & & & 1 & 0.159 & $0.493^{* \dagger}$ & 0.403 & 0.149 & -0.252 & $-0.524^{* \dagger}$ & 0.182 \\
\hline $\begin{array}{l}\text { Handgrip } \\
\text { strength } \\
{[\mathrm{N}]}\end{array}$ & $\left|r_{s}\right|$ & & & & & & 1 & 0.424 & $0.834 * *$ & $-0.505^{* \dagger}$ & -0.249 & 0.431 & $0.675^{* * \dagger}$ \\
\hline MP [W] & $r_{s}$ & & & & & & & 1 & 0.400 & 0.305 & 0.351 & -0.227 & 0.045 \\
\hline $\mathrm{PP}[\mathrm{W}]$ & $r_{s}$ & & & & & & & & 1 & -0.284 & -0.369 & 0.256 & $0.764 * * \neq$ \\
\hline tPP [s] & $r_{s}$ & & & & & & & & & 1 & $0.542 * * \dagger$ & -0.132 & -0.310 \\
\hline \begin{tabular}{|l} 
rMP \\
{$[\mathrm{W} / \mathrm{kg}]$}
\end{tabular} & $r_{s}$ & & & & & & & & & & 1 & 0.210 & -0.323 \\
\hline $\begin{array}{l}\mathrm{rPP} \\
{[\mathrm{W} / \mathrm{kg}]}\end{array}$ & $r_{s}$ & & & & & & & & & & & 1 & $0.507 * \dagger$ \\
\hline FI [W/s] & $r_{s}$ & & & & & & & & & & & & 1 \\
\hline
\end{tabular}

$* p<0.05 ; * * p<0.01 ;^{\dagger}-$ moderate correlation; ${ }^{*}$ - strong correlation; WBW - whole-body weight; A-BMI - amputation-adjusted BMI; FAT \% - percentage of body fat; MP - mean power; PP - peak power; tPP - time to achieve peak power; rMP - relative mean power; rPP - relative peak power; FI - fatigue index.

\section{Discussion}

The study first aimed to determine anaerobic performance in male AF players in terms of the type and the level of limb impairment, playing position, and anthropometric parameters, comparing the findings with reference values for athletes with lower-limb impairments, and, secondly, to verify whether there was a relationship between parameters in the WAnT and the handgrip test, and both aims were achieved.

First, no differences in anaerobic performance results (PP, MP, rPP, rMP, tPP, FI) were found between the groups considering the level of leg length. There was a difference in FI between the limb deficiency group and the leg length difference group ( $p=0.026$, medium ES). FI is an additional parameter that assesses level of decreasing of power in the WAnT. One of the most important parameters in an athlete anaerobic performance analysis is the curve in the WAnT. Therefore, we suggest analysing MP and PP as well as the curve in the WAnT to interpret anaerobic performance of an athlete.
In the next part of the current study, anaerobic performance results were compared between defenders and forwards. No studies related to anaerobic performance assessment of AF players with the use of the WAnT and in general anaerobic-character laboratory tests were found. The findings of the present study make it possible to pre-define the characteristics of forwards and defenders based on anaerobic performance and its reference values establish for athletes with lower-limb impairments and anthropometric parameters [20]. Defenders demonstrated significantly higher PP results in the WAnT ( $p=0.027$, large ES), and they were significantly taller and heavier, whereas forwards obtained significantly higher relative power results (rMP and rPP, $p=0.049$, large ES). It is worth underlying that, in the case of rPP, the positive effect occurs when greater WBW results in higher rPP values. However, the increased amount of FAT \% explained by greater WBW does not exert a positive influence on rPP, which is confirmed in this study ( $r=$ $-0.516, p<0.05)$. It is recommended to include more anaerobic exercises to the current defender's training. It may cause better rMP and rPP results with their 
actual high amount of FAT \% and WBW which could bring their results closer to forwards' relative power results. On the other hand, WBW loss and the inclusion of a specific diet, should not be the only task to achieve higher relative power values. Body composition control (ratio of FAT \% to muscle mass) and anaerobic exercises in the current training plan should be the next step for these players to have higher anaerobic performance abilities. Thanks to this, defenders may be more dynamic in the AF game.

Secondly, defenders and forwards were compared considering anthropometric parameters. In case of defenders, the WBW, FAT \% and A-BMI were statistically higher than in forwards $(p<0.05$, large ES). People with limb loss might not appreciate their health status related to body weight while using BMI [39]. The ratio of body weight and body height might indicate a misleading image if a part of a lost limb is not taken into consideration. Defining A-BMI can facilitate monitoring body size, simultaneously supporting (similarly to BMI) the evaluation a risk of overweight-related diseases (e.g., atherosclerosis, ischemic heart disease) as well as to make dietary decisions. The described-above factors might influence sport performance.

The literature demonstrates that midfielder AF players had significantly lower WBW $(p=0.520)$ than other players and had significantly lower FAT \% than defenders $(p=0.011)$ [30]. In another study, though, AF midfielders had the greatest WBW (their WBW was significantly higher than that of strikers; $p<0.05$ ) and had the highest FAT \% in relation to strikers and defenders [4]. However, Simim [30] noted that defenders had the greater WBW than other players, which is in line with the findings of the current study. These differences in WBW in the aforementioned studies may stem from different ways of grouping the players according to their positions (only defenders and forwards or defenders, strikers and midfielders). Picking out players for positions may be related not only to their anthropometric predisposition but also to their technical and cognitive abilities that are important especially when players do actions with a ball or their previous experience in soccer [29]. As far as detailed data is concerned, the study by da Silva et al. [4] revealed that midfielders, as the players with the highest FAT \%, demonstrated the values of FAT \% remarkably similar to the indicators obtained by defenders in the current study as well as in the investigation by Simim et al. [30], i.e., approximately $18 \%$. It is worth noting that in this study, the near-infrared (NIR) spectroscopy was used to estimate FAT \%. In other studies, different types of skinfold callipers and different methods of skinfold thickness were used [4], [9], [27], [30]. Unfortunately, among people with amputations it is difficult to properly estimate body composition (FAT \%, WBW). A-BMI could be a solution, however, we have not found studies where A-BMI was used among amputee athletes. For these reasons, the results from different studies cannot be easily compared. Currently, based on available literature and present study's results, the characteristics of AF players in terms of body composition cannot be clearly defined. In future studies, authors should consider using A-BMI. It is recommended to find appropriate tools to assess FAT \% and others body composition parameters in amputee population.

Thirdly, anaerobic performance results of AF players were presented in comparison with reference values for athletes with lower-limb impairment described by Molik [20]. 22 out 23 players reached at least "average" rPP and 16 out 23 reached at least "average" rMP. 9 out of 23 players were "excellent" and "elite" in rPP. Most of the study participants (19 out of 23) achieved results from "average" to "elite" in PP. MP was the worst parameter, in which 13 out 23 players were "below average". These observations confirmed that a greater role of PP compared to MP in AF stems from the specificity of this game (players start to run and stop to kick the ball, move forward quickly, change movement directions rapidly, etc.), which is reflected in PP value in the WAnT. Additionally, the results of rPP parameters stood out in players' anaerobic performance since it achieved better outcomes than other parameters, which were categorized as "general: or "average" or "below average" level. Players divided into the type of impairment groups had "above average" rPP results as well as players with AKA. Moreover, "excellent" rPP score achieved forwards while defenders scored "average" in rPP but "above average" in PP. rPP reflects the specificity and characteristic features of AF game with fast and dynamic moves, changing directions and accelerations in a few seconds. Not only body composition and physical fitness but also cognitive performance is important in sport. A combination of cognitive and physical training adjusts athlete's performance and can help coaches to scout for talents [29]. For future studies, it seems to be valuable to add psychological and cognitive tests for analysis.

On the other hand, there is no classification system referring to the types of lower-limb impairment in AF, unlike in other Para sports. For instance, in wheelchair basketball there is a classification system in which athletes with longer stumps are allocated higher functional sport classes, in athletics, there are different sport 
classes for lower and upper limb amputees, while in swimming, athletes are divided according to different sport classes because of the level of amputation of upper/lower limb/s [12], [13]. What is significant about classification systems in Para sports is that the aim of sport classification is to minimize the impact of impairments on performance and to ensure the success of an athlete is determined by skills, physical fitness, tactical ability, and mental focus [12], [13]. Considering the above-mentioned examples of classification in Para sports and a general aim of classification, it appears that in AF, players might be divided because of the level of leg length or the type of impairment. However, in our study there were no differences in anaerobic performance parameters between groups in terms of the level of leg length and the type of impairment and (except for FI). It might be assumed that there is no need to create sport classes in AF or the type of impairment. Contrary to that, a functional level of impaired lower limb might be a reason for the division of the players into groups both for researchers and for playing. More and wider analysis are needed augmented with bio- and kinematic analyses which could complement this hypothesis. Nevertheless, future studies in evidence-based classification in AF will be required by the International Paralympic Committee if AF would like to apply to be a part of the Paralympic movement [12], [13].

In the last part of this study, correlations between variables demonstrated some practical aspects of this investigation. AF players with greater WBW have a lower ability to maintain power (high FI value) and may become exhausted faster (moderate correlation was found between age and FI; $r_{s}=0.487$, $p<0.05$ ). It might constitute a good prompt for coaches that keeping appropriate players' WBW as well as their proper body composition is important in anaerobic performance. It allows performing soccertype moves and may lead to winning a game. In the analysis, there was a strong correlation between handgrip strength results and PP $\left(r_{s}=0.805, p<0.01\right)$. This correlation could indicate that handgrip test might be used to assess PP indirectly like it was used by other authors in people with impairments and alebodied, i.e., AF players, children with spina bifida, youth handball players and adult wheelchair basketball players [17], [18], [25], [28], [38]. However, we do not recommend this indirect assessment of PP because there were no correlations between handgrip strength and relative parameters in the WAnT (rPP, rMP) in this study. Then, the handgrip test bases on isometric contraction muscle strength, and time of handgrip test is short ( $2-3$ seconds) in comparison to the WAnT (30 seconds). Additionally, in the WanT a major part of body is involved while during the handgrip test only one hand is involved. Despite the correlations identified, it is difficult to count this test as an anaerobic performance assessment test for upper limbs in AF group.

\subsection{Limitations and recommendations for future studies}

In future examinations, more participants from the elite level of AF should be included and players should be divided more specifically according to playing position in the game (strikers, midfielders, defenders). However, there are not many elite players with many years of experience in AF. It is understandable that the study group was not large enough to be a valid representation, the most elite athletes from Poland were invited to participate in the study. The participants were divided by impairment type into unequal groups (7 vs. 16). It is acknowledged that having the same number of people in both groups would be the best course of action, but there were only so many athletes to work with.

Considering that AF has not been a Paralympic sport yet and applies to be a member of Paralympic movement, evidence for players' division or no division into sport classes is sought in future classification studies. It is very important for this sport to conduct a research regarding an evidence-based classification concept if they were included in the Paralympic movement as a Paralympic sport.

In this study, the Futrex device using NIR was applied to estimate FAT \%. We found two studies where the Futrex device was used among athletes with impairment [Molik, Basar]. We have not found studies on the incorrect use of this tool in people with impairment. Nevertheless, researchers should be careful in the results interpretation. Our recommendation is to find a reliable method to assess body components of amputee people.

In our opinion an important part of the originality of this study is that we used the WAnT among AF players. It is a relatively new sport and we recommend continuing analyses of aerobic and anaerobic performance in AF players. The WAnT on the arm-crank ergometer or on the cycle ergometer are widely known tests to assess anaerobic performance in ablebodied and in disability sport. The specificity of AF players probably requires checking the involvement of muscles during match performance, in accordance with anaerobic performance of not only upper limbs 
but also lower limbs. Additionally, there are no sportspecific anaerobic tests for AF. As it was mentioned, short-terms efforts in the game lasted a few seconds, full actions more, but further analyses are needed. Therefore, the next recommendation is to create more specific, high-intensity intermittent laboratory and/or field-based tests for AF players that would involve upper and/or lower limbs. Furthermore, field-based tests for AF players should be specific and ought to consider similar efforts as movements in the game.

\section{Conclusions}

Specific character of amputee football (AF) requires a high level of power from players, especially relative peak power values and indicate high-intensity intermittent (stop-go) characteristics of AF. Coaches should focus on adapting their players to anaerobic movements during everyday practice and training.

Body composition (the ratio of lean body mass to FAT \%) is important for AF defenders and forwards to be more dynamic and generate more relative peak power (rPP) in short time (explosive power). Coaches should cooperate with dieticians to monitor player's body composition to have better results.

The 30-second Wingate Anaerobic Test (the WAnT) can be used to assess anaerobic performance in AF players. However, sport-specific anaerobic performance laboratory tests and-field-based tests using in indirect upper limbs' peak power monitoring may be beneficial for coaches in their work.

\section{Funding}

This work was supported by the Ministry of Science and Higher Education in the year 2021 under Research Group no. 4 at Józef Piłsudski University of Physical Education in Warsaw "Physical activity and sports for people with special needs".

\section{Acknowledgements}

We would like to thank all the study participants for their interest in research and inquisitiveness in research discussions about their results in tests.

\section{References}

[1] Bowling A., Research methods in health. Investigating health and health services, Open University Press, 2002.
[2] Brasile F.M., Performance evaluation of wheelchair athletes: more than a disability classification level issue, APAQ, 1990, 7 (4), 289-297.

[3] COHEN J., Statistical power analysis for the behavioral sciences, Lawrence Erlbaum Associates, 1988.

[4] Da Silva G.A.I., Ribeiro B.G., DE ABreu S.E., Nutritional profile of the Brazilian Amputee Soccer Team during the precompetition period for the world championship, Nutrition, 2006, 22 (10), 989-995.

[5] De Lira C.A.B., Vancini R., Minozzo F.C., Sousa B.S., Dubas J.P., ANDrade M.S., SteinBerg L.L., Da Silva A.C., Relationship between aerobic and anaerobic parameters and functional classification in wheelchair basketball players, Scand. J. Med. Sci. Sports, 2009, 20 (4), 638-643.

[6] Federazione Italiana Sport Paralimpici e Sperimentali, World Amputee Football Federation Laws and Rules Governing Amputee Football Play and Players, 2017, https://fispes.it/kp/ uploads/laws-of-the-game-2017.pdf (Accessed: 25 April 2020).

[7] FuKuda D.H., Wray M.E., Kendall K.L., SMith-Ryan A.E., STOUT J.R., Validity of near-infrared interactance (FUTREX 6100/XL) for estimating body fat percentage in elite rowers, Clin. Physiol. Funct. Imaging, 2017, 37 (4), 456-458.

[8] GöKTEPE S.A., Energy system in sports, Amputee Sports for Victims of Terrorism, IOS Press, 2007, 24-31.

[9] GÜÇhan Z., Bayramlar K., ERGun N., Determination of the effects of playing soccer on physical fitness in individuals with transtibial amputation, J. Sport Med. Phys. Fit., 2017, 57 (6), 879-886.

[10] Hutzler Y., Vanlandewijck Y., Vlierberghe V., Anaerobic performance of older female and male wheelchair basketball players on a mobile wheelchair ergometer, APAQ, 2000, 17 (4), 450-465.

[11] InBAR O., BAR-OR O., SKInNER J., The Wingate Anaerobic Test, Human Kinetics, 1996.

[12] International Paralympic Committee, Explanatory guide to Paralympic classification. Paralympic summer sports, 2015, https:/www.paralympic.org/sites/default/files/document/150915 170806821_2015_09_15\%2BExplanatory\%2Bguide\%2BClassif ication_summer\%2BFINAL\%2B 5.pdf (Accessed: 22 April 2020).

[13] International Paralympic Committee, Explanatory guide to Paralympic Classification. Paralympic winter sports, 2016, https://www.paralympic.org/sites/default/files/document/160 $211172359750 \quad 2016 \% 2 \mathrm{~B} 02 \% 2 \mathrm{BWinter} \% 2 \mathrm{BExplanatory} \% 2$ BGuide\%2B.pdf (Accessed: 22 April 2020).

[14] Lijewski M., Burdukiewicz A., Pietraszewska J., Stachoń A., Andrzejewska J., Chromik K., Anthropometric and strength profiles of professional handball players in relation to their playing position - multivariate analysis, Acta Bioeng. Biomech., 2019, 21 (4), 147-155.

[15] Maehana H., Miyamoto A., Koshiyama K., YanagiYa T.T., Yoshimura M., Profile of match performance and heart rate response in Japanese amputee soccer players, J. Sport Med. Phys. Fit., 2018, 58 (6), 816-824.

[16] MarszaleK J., Molik B., Gomez M.A., Skucas K., LencSe-Mucha J., Rekowski W., Pokvytyte V., Rutkowska I., KAZMIERSKA-KOWALEWSKA K., Relationships between anaerobic performance, field tests and game performance of sitting volleyball players, J. Hum. Kinet., 2015, 48, $25-32$.

[17] Marszalek J., Kosmol A., Morgulec-Adamowicz N., Mroz A., Gryko K., Klavina A., Skucas K., Navia J.A., MOLIK B., Laboratory and non-laboratory assessment of an- 
aerobic performance of elite male wheelchair basketball athletes, Front Psychol., 2019, 10, 514.

[18] Marszalek J., Kosmol A., Morgulec-Adamowicz N., Mroz A., Gryko K., MoliK B., Test-retest reliability of the newly developed field-based tests focuses on short time efforts with maximal intensity for wheelchair basketball players, Advances in Rehabilitation, 2019, 33 (1), 23-27.

[19] Miyamoto A., Maehana H., YanagiYa T., The relationship between sprint speed and sprint motion in amputee soccer players, EUJAPA, 2019, 12 (9), 1-9.

[20] MoliK B., Anaerobic performance and efficiency in team games versus classification of athletes with disabilities, Habilitation thesis, Józef Piłsudski University of Physical Education, Warsaw, 2010.

[21] Molik B., Kosmol A., Laskin J.J., Skucas K., Bida U., Relationship between functional classification levels and anaerobic performance of wheelchair basketball players, Res. Q. Exerc. Sport, 2010, 81 (1), 69-73.

[22] Molik B., Laskin J.J., Kosmol A., MarszaleK J., MorguleC-AdAmOWICZ N., Frick T., Relationships between anaerobic performance, field tests, and functional level of elite female wheelchair basketball athletes, Hum. Mov., 2013, 14 (4), 366-371.

[23] Morgulec-Adamowicz N., Kosmol A., Molik B., Yilla A., LASKIN J.J., Aerobic, anaerobic, and skill performance with regard to classification in wheelchair rugby athletes, Res. Q. Exercise Sport, 2011, 82 (1), 61-69.

[24] Nindl B.C., Mahar M.T., Harman E.A., Patton J.F., Lower and upper body anaerobic performance in male and female adolescent athletes, Med. Sci. Sports Exerc., 1995, 27 (2), 235-241.

[25] Nowak A.M., Molik B., Marszalek J., Anaerobic performance among children with spina bifida, J. Sports Med. Phys. Fitness, 2020, 60 (1), 132-139.

[26] NowaK A.M., Match performance in Polish amputee soccer Extra Ligue - a pilot study, Advances in Rehabilitation, 2020, 34 (2), 16-25.

[27] ÖZkan A., Kayihan G., KöKlÜ Y., ERgun N., Koz M., ERSÖZ G., Dellal A., The relationship between body composition, anaerobic performance and sprint ability of amputee soccer players, J. Hum. Kinet., 2012, 35 (1), 141-146.

[28] RDZANeK J., Michalska A., WyChOWANSKi M., TARGOSInSKi P., Assessment of handgrip strength in young handball players aged 9-16, Advances in Rehabilitation, 2019, 33 (2), $13-19$.

[29] Scharfen H.-E., Memmert D., The relationship between cognitive functions and sport specific motor skills in elite youth soccer players, Front Psychol., 2019, 10, 1-10.

[30] Simim M.A.M., Silva B.V.C., Marocolo M., Mendes E.L., De Mello M.T., Da Mota G.R., Anthropometric profile and physical performance characteristic of the Brazilian amputee football (soccer) team, Revista de Educacao Fisica, 2013, 19 (3), 641-648.

[31] Simim M.A., Bradley P.S., Da Silva B.V., Mendes E.L., De Mello M.T., Marocolo M., Da Mota G.R., The quantification of game-induced muscle fatigue in amputee soccer players, J. Sport Med. Phys. Fit., 2017, 57 (6), 766-772.

[32] Simim M., Da Mota G., Marocolo M., Da Silva B., De Mello M.T., Bradley P., The demands of amputee soccer impair muscular endurance and power indices but not match physical performance, APAQ, 2018, 35 (1), 76-91.

[33] Stølen T., Chamari K., Castagna C., Wisløff U., Physiology of soccer: an update, Sports Med., 2005, 35 (6), 501-536.

[34] Tatar Y., Gercek N., Ramazanoglu N., Gulmez I., UzuN S., Sanli G., Karagozoglu C., CotuK H.B., Load distribution on the foot and Lofstrand Crutches of amputee football players, Gait and Posture, 2018, 64, 169-173.

[35] Thys H., Willems P.A., SAels P., Energy cost, mechanical work and muscular efficiency in swing-through gait with elbow crutches, J. Biomech., 1996, 29, 1473-1482.

[36] Tzamaloukas A.H., Patron A., Malhotra D., Body mass index in amputees, J. Parenter Enteral Nutr., 1994, 18 (4), 355-358.

[37] Vlassoli T.O., Zafirova B., Orovcanec N., Poposka A., MURTEZANI A., KRASINIQI B., Energy expenditure and walking speed in lower limb amputees: a cross sectional study, Ortop. Traumatol. Rehabil., 2014, 16 (4), 419-426.

[38] Wieczorek M., Wilinski W., StruZik A., Rokita A., Hand grip strength vs. sprint effectiveness in amputee soccer players, J. Hum. Kinet., 2015, 48, 133-139.

[39] Wong C.K., Wong R.J., Standard and amputation-adjusted body mass index measures. Comparison and relevance to functional measures, weight-related comorbidities, and dieting, Am. J. Phys. Med. Rehabil., 2007, 96 (12), 912-915.

[40] YAZICIOGLU K., The rules of amputee football, Amputee Sports for Victims of Terrorism, IOS Press, 2007, 94-99. 